\title{
Overcoming sparseness of biomedical networks to identify drug repositioning candidates
}

\author{
Aleksandar Poleksic \\ Department of Computer Science, University of Northern lowa, Cedar Falls, IA 50613
}

\begin{abstract}
Modeling complex biological systems is necessary to understand biochemical interactions behind pharmacological effects of drugs. Successful in silico drug repurposing requires a thorough exploration of diverse biochemical concepts and their relationships, including drug's adverse reactions, drug targets, disease symptoms, as well as disease associated genes and their pathways, to name a few. We present a computational method for inferring drug-disease associations from complex but incomplete and biased biological networks. Our method employs the compressed sensing technique to overcome the sparseness of biomedical data and, in turn, to enrich the set of verified relationships between different biomedical entities. We present a strategy for identifying network paths supportive of drug efficacy as well as a computational procedure capable of combining different network patterns to better distinguish treatments from non-treatments. The data and programs are freely available at http://bioinfo.cs.uni.edu/AEONET.html.
\end{abstract}

\section{Introduction}

Recent decades have seen a sharp increase in spending on drug development in US and the rest of the world. The number of drugs approved per 1 billion dollars invested in R\&D has steadily declined since $1950^{1}$, with the estimates of the average cost to bring a new drug to the market approaching 1.7 billion ${ }^{2}$. Reversing the trend of the stagnant and diminishing drug approval rates requires adoption of new approaches to drug discovery. To address the toxicity and lack of efficacy of candidate drugs, researchers are increasingly looking for therapeutics that act on multiple targets pertaining to single or multiple disease pathways. Such a broader approach to the development of pharmaceutical agents, coined as polypharmacology ${ }^{3,4}$, often necessitates the use of multiple chemicals acting on different targets for the treatment of complex diseases. Polypharmacology is the main principle behind drug repurposing, which aims to identify new uses for the existing drugs. Drug repurposing accelerates the discovery process by bypassing most of the pre-clinical work and trials required for drug approval.

Computational methods provide crucial support for drug repurposing. Modern algorithms increasingly rely on the network representation of a biological system to discover drug-disease, drug-gene, disease-gene, and other relationships between biomedical concepts. A simple biological system can be viewed as a homogenous network in which the nodes represent biological entities and edges represent the relationships (associations or interactions) between those entities. An example is the protein interaction network, in which the nodes represent proteins and edges signify PPIs.

While homogenous network can be used to model relationships between entities from any pair of domains, the accumulating scientific evidence suggests that better understanding of a biological system as a whole is necessary to overcome the limited performance of methods that only take account of one or two types of biological data. Complex biological systems can be viewed as heterogeneous networks in which the nodes are grouped into different layers (domains), such as the compound layer, protein layer, disease layer, etc. The nodes within each layer are interconnected and connected to the nodes from other layers ${ }^{5}$.

Heterogeneous networks provide new avenues for research into in silico drug repurposing ${ }^{6,7,8,9}$. Zeng et al. used a multi-modal autoencoder on a heterogeneous network to 
learn drug features and, in turn, prioritize drug repurposing candidates ${ }^{10}$. Chen et al. developed an efficient algorithm for detecting missing cross-layer links in a heterogeneous network by leveraging the observed cross-layer dependency with the within-layer topology ${ }^{11}$. Guney et al. developed a drug-disease proximity measure to quantify the network-based associations between drugs and disease proteins ${ }^{12}$. Cheng et al. proposed a systems pharmacology-based procedure that integrates molecular pharmacoepidemiologic and network-based approaches to quantify interplay between drug targets and disease proteins ${ }^{13}$.

Perhaps the most comprehensive biological network to date is HetioNet $^{14}$. This heterogeneous network, compiled from public resources ${ }^{15-54}$, consists of 47,031 nodes grouped into 11 domains and 2,250,197 relationships (edges) classified into 24 categories (Fig. 1).

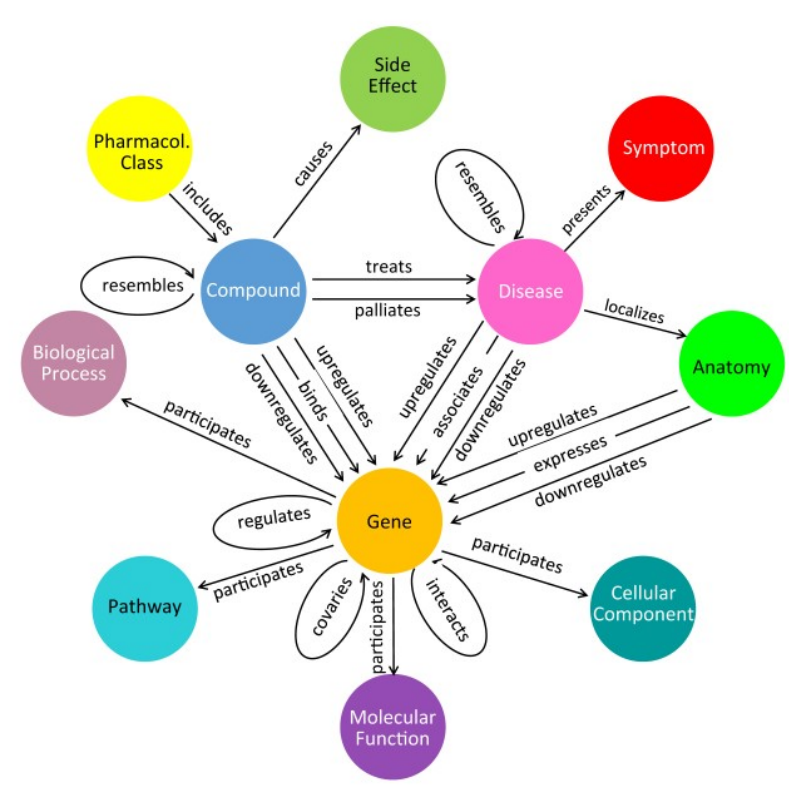

Relationships (meta-edges)

\begin{tabular}{|l|l|}
\hline Anatomy-downregulates-Gene & AdG \\
\hline Anatomy-expresses-Gene & $A e G$ \\
\hline Anatomy-upregulates-Gene & $A u G$ \\
\hline Compound-resembles-Compound & CrC \\
\hline Compound-causes-Side Effect & CcSE \\
\hline Pharmacologic Class-includes-Compound & PCiC \\
\hline Compound-treats-Disease & CtD \\
\hline Compound-palliates-Disease & CpD \\
\hline Compound-binds-Gene & CbG \\
\hline Compound-downregulates-Gene & CdG \\
\hline Compound-upregulates-Gene & CuG \\
\hline Disease-resembles-Disease & DrD \\
\hline Disease-localizes-Anatomy & DIA \\
\hline Disease-presents-Symptom & DpS \\
\hline Disease-associates-Gene & DaG \\
\hline Disease-downregulates-Gene & DdG \\
\hline Disease-upregulates-Gene & DuG \\
\hline Gene-covariates-Gene & GcG \\
\hline Gene-interacts-Gene & GiG \\
\hline Gene-regulates-Gene & GrG \\
\hline Gene-participates-Pathway & GpPW \\
\hline Gene-participates-Biological Process & GpBP \\
\hline Gene-participates-Molecular Function & GpMF \\
\hline Gene-participates-Cellular Component & GpCC \\
\hline
\end{tabular}

Fig 1. HetioNet

In the project Rephetio, Himmelstein et al. demonstrated the advantage of utilizing HetioNet patterns to reconstruct known and predict new drug-disease associations ${ }^{14}$. Rephetio is capable of identifying multiple network paths that explain therapeutic efficacy of different drugs on different diseases. The method uses logistic regression to fine-tune the weights of different network paths that connect compounds to diseases, including transitive paths that traverse multiple relations, such as, for example, Compound-binds-Gene-participates-Pathway-participates-Geneassociates-Disease. (The meta-path in this example represents any path from a compound node $c$ to a disease node $d$ with the property that $c$ binds a gene $g_{1}$ which participates in the same pathway as gene $g_{2}$, which is associated to the disease $d$.) The aggregate probability over selected paths is used to measure the strength of each drug-disease association.

While the novel network-based approach used by Himmelstein et al. is useful in gaining insight into the drug mechanism of action, the accuracy of Rephetio is highly dependent on the available network data. Here we present a computational method AeoNet, which is capable of identifying network patterns that explain drug efficacy in context of sparse, biased and incomplete networks. Our algorithm can be viewed as a two-step process.

First, we use the compressed sensing technique ${ }^{55,56,57}$ to enrich the set of network edges by inferring all missing inter-domain and cross-domain relationships. In the second step, we integrate the likelihoods of all possible paths connecting each drug with each disease. We use three external benchmarks to demonstrate that our approach consistently increases the accuracy of drug-disease association prediction. 
The rest of this paper is organized as follows. In section 2.1 we provide a brief overview of compressed sensing. Section 2.2 describes a simple procedure for constructing different network paths that connect drugs to diseases. Section 2.3 presents a strategy for selecting a set of the most informative paths, while section 2.4 presents a simple method for aggregating drugdisease association probabilities along those paths. The Results section summarizes the performance of our method on the external validation sets.

\section{Methods}

\subsection{Inferring missing HetioNet relationships}

We use a variant of the matrix completion technique, referred to as "compressed sensing", to enrich the set of edges between any two HetioNet domains connected by a meta-edge.

Compressed sensing is similar in spirit to dual regularized one-class collaborative filtering (OCCF) algorithm ${ }^{58}$. However, in contrast to OCCF, our method is based on logistic matrix factorization ${ }^{59}$ and thus has a more explicit statistical foundation.

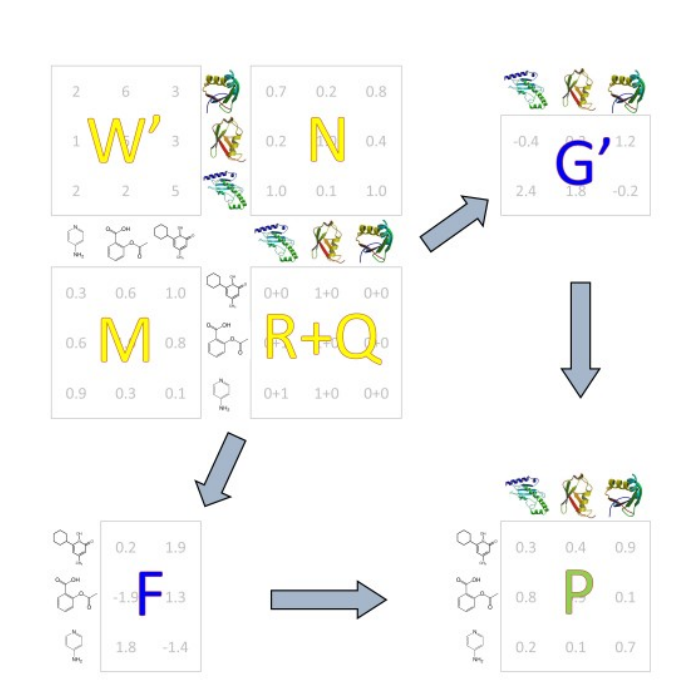

Fig. 2. Compressed sensing algorithm. R: input matrix of drug-target associations; Q: impute matrix; W: weight matrix; M: drug similarity matrix; $\mathrm{N}$ : target similarity matrix; $\mathrm{F}$ : latent drug preferences; G: latent target preferences; P: output matrix of drug-target interaction probabilities.
The compressed sensing algorithm

In what follows, we illustrate how this procedure works in an example setting of drug-target interaction prediction. The same algorithm is applied to predict relationships between entities that belong to other directly connected biological domains.

There are two scientific premises behind our method. First, we assume that the matrix of drug-target association (that we wish to recover) is of small rank $k \ll \min \{n, m\}$, where $n$ and $m$ denote the total number of drugs and genes, respectively. Our algorithm (Fig. 2) can be viewed as the low-rank completion of the input (noisy and incomplete) drug-target association matrix $R=$ $\left(r_{i, j}\right)$, defined as

$$
r_{i, j}=\left\{\begin{array}{lc}
1 & \text { if drug } i \text { interacts with target } j \\
0 & \text { otherwise }
\end{array}\right.
$$

Our second assumption is that similar drugs interact with similar targets. Given the relationship

matrix $R$, the pairwise drug similarity matrix $M$, and the pairwise target similarity matrix $N$, the algorithm computes the matrices $F$ and $G$ of drugs' and targets' "latent" preferences (Fig. 2). Specifically, the $i^{\text {th }}$ row of $F$ represents the latent vector representation of the drug $i$ while the $i^{\text {th }}$ row of $G$ represents the latent vector representation of target $i$. The matrices $F$ and $G$ are found by minimizing the loss function

$$
\begin{aligned}
\sum_{i, j} w_{i, j} & \left\{\ln \left(1+e^{f_{i} g_{j}^{\prime}}\right)-\left(r_{i, j}+q_{i, j}\right) f_{i} g_{j}^{\prime}\right\}+\lambda_{r}\left(\|F\|_{2}^{2}+\|G\|_{2}^{2}\right)+ \\
& +\lambda_{M} \operatorname{tr}\left(F^{\prime}\left(D_{M}-M\right) F\right)+\lambda_{N} \operatorname{tr}\left(G^{\prime}\left(D_{N}-N\right) G\right)
\end{aligned}
$$

In (1) above, the prime symbol denotes matrix transpose, \|\|$_{2}$ stands for the Frobenius norm, and $t r$ denotes the matrix trace. We use $f_{i}$ and $g_{j}$ to denote the $i^{\text {th }}$ row of $F$ and $j^{\text {th }}$ row of $G$, 
respectively. The matrix $D_{M}$ is the diagonal "degree matrix" of $M$ in which the element $d_{i, i}$ represents the sum of the elements in the $i^{\text {th }}$ row of $M$. The (optional) impute values $q_{i, j}$ can be specified by expert users who wish to supplement the set of relationships $R$, while the entries $w_{i, j}$ of the weight matrix $W$ reflect the user's confidence in the elements of the interaction matrix $R+Q$. The lambdas $\left(\lambda_{r}, \lambda_{M}\right.$, and $\left.\lambda_{N}\right)$ are optimizable parameters. The matrix of probabilities of drug-target interactions is computed as $P=\exp \left(F G^{\prime}\right) /\left(1+\exp \left(F G^{\prime}\right)\right)$. The latent preferences of drugs with no known interacting targets and targets with no known interacting drugs are derived with a variant of the weighted profile method. For technical details, we refer the reader to our published work ${ }^{55,56,57}$.

\subsection{Exploring different paths connecting drugs to diseases}

We compute drug-disease association probabilities separately along each path between the drug layer and the disease layer.

\section{$N=D p s D$}

\section{CcSEcCtDpSpD}

$$
M=\operatorname{CseC} \quad R
$$

Fig. 3. Assigning pathdependent matrices $R, M$ and $N$.
The variety of paths between two layers gives rise to a set of diverse similarity matrices $M$ and $N$ that can be specified as input to our compressed sensing algorithm. To derive side information specific to a particular network path $p$, we split $p$ into three subpaths, $p_{1}, p_{2}$, and $p_{3}$. The sub-path $p_{1}$ is a cycle that starts and ends at the first layer and uniquely defines a similarity matrix $M$. The subpath $p_{2}$ consists of a single edge that connects the first layer to the second layer and corresponds to the relationship itself (matrix $R$ ). Finally, the sub-path $p_{3}$, determining the side information $N$, is a cycle that starts and ends at the second layer.

For instance, the path $p=C r C t D r D$ (Compound-resembles-Compound-treatsDisease-resembles-Disease) can be represented as $p=p_{1} p_{2} p_{3}$, where $p_{1}=\operatorname{CrC}$, $p_{2}=C t D$, and $p_{3}=D r D$. The drug-disease association probabilities along this path are derived by running the compressed sensing algorithm on the input drug similarity matrix $M=C r C$ (Compoundresembles-Compound), the association matrix $R=C t D$ (Compound-treats-

Disease), and the disease similarity matrix $N=\operatorname{DrD}$ (Disease-resembles-Disease). The same idea is extended to derive $M, R$, and $N$ along longer paths. For instance, as illustrated in Fig. 3, the drug-disease probabilities along the path CcSEcCtDpSpD (Compound-causes-Side Effect-causes-Compound-treats-Diseasecauses-Symptom-causes-Disease) are computed by running the compressed sensing algorithm on the input matrices $M$

Table 1. Different drug, gene, and disease similarity matrices.

\begin{tabular}{c|c}
\hline $\begin{array}{c}\text { Compound } \\
\text { Similarity Matrix }\end{array}$ & Derived from HetioNet Relationship \\
\hline CrC & Compound-resembles-Compound \\
\hline CseC & Compound-causes-Side Effect (CcSE) \\
\hline CpcC & $\begin{array}{c}\text { Pharmacologic Class-includes- } \\
\text { Compound (PCiC) }\end{array}$ \\
\hline CsC & Average of CseC and CpcC \\
\hline $\begin{array}{c}\text { Disease } \\
\text { Silarity Matrix }\end{array}$ & Derived from HetioNet Relationship \\
\hline DrD & Disease-resembles-Disease \\
\hline DpsD & Disease-presents-Symptom (DpS) \\
\hline DlaD & Anatomy (DIA) \\
\hline DsD & Average of DpsD and DlaD \\
\hline Gene Similarity & Derived from HetioNet Relationship \\
\hline GbpG & Biological Process (GpBP) \\
\hline GmfG & Molecular Function (GpMF) \\
\hline GccG & Cellular Component (GpCC) \\
\hline$G p w G$ & Pathway (GpPW) \\
\hline$G s G$ & Average of GbpG, GmfG, GccG, \\
GpwG, GiG, GrG, GcG
\end{tabular}
$=C c S E c C, R=C t D$, and $N=D p S p D$, where $C c S E c C$ (abbreviated as CseC) represents the matrix of pairwise similarities of drugs' side-effect profiles and $D p S p D$ (or short $D p s D$ ) represents the matrix of pairwise similarities of disease' symptom profiles (Table 1, Fig. 3). More specifically, given compounds $i$ and $j$, we set the element of the matrix $C s e C$ at the position $(i, j)$ to the Jaccard similarity of the rows $i$ and $j$ of the CCSE matrix (Compound-causes-Side 
Effect). The disease similarity matrix $N=D p s D$ is specified in a similar manner, by computing Jaccard similarities of diseases' symptom profiles stored in the relationship matrix $D p S$ (Disease-presents-Symptom).

In theory, $p_{1}$ or $p_{3}$ (or both) can be of length zero, indicating the lack of side information. In those cases, the input similarity matrices are simply set to the identity matrices. For instance, if $p=\operatorname{CrCtD}$, then $M=C r C, R=C t D$, and $N=I$ (no drug side information).

Table 2. Classification accuracy of different network patterns (three rounds of 3-fold CV). The paths with highest AUC scores are shown in bold.

\begin{tabular}{l|l|l}
\hline Path & AUPR & AUC \\
\hline CrCtDsD & $\mathbf{0 . 2 1 1 ( 0 . 0 2 1 )}$ & $\mathbf{0 . 9 1 4 ( 0 . 0 0 9 )}$ \\
\hline CpcCtDsD & $\mathbf{0 . 2 5 5 ( 0 . 0 1 2 )}$ & $\mathbf{0 . 9 2 0 ( 0 . 0 0 5 )}$ \\
\hline CseCtDsD & $\mathbf{0 . 3 5 0 ( 0 . 0 1 6 )}$ & $\mathbf{0 . 9 4 2 ( 0 . 0 0 8 )}$ \\
\hline CsCtDrD & $\mathbf{0 . 3 0 2 ( 0 . 0 0 7 )}$ & $\mathbf{0 . 9 3 8 ( 0 . 0 0 1 )}$ \\
\hline CsCtDIaD & $\mathbf{0 . 2 9 8 ( 0 . 0 1 4 )}$ & $\mathbf{0 . 9 3 8 ( 0 . 0 0 6 )}$ \\
\hline CsCtDpsD & $\mathbf{0 . 3 0 1 ( 0 . 0 1 1 )}$ & $\mathbf{0 . 9 3 6 ( 0 . 0 0 2 )}$ \\
\hline CsCbGsGaDsD & $\mathbf{0 . 0 2 9}(\mathbf{0 . 0 0 0})$ & $\mathbf{0 . 8 2 4 ( 0 . 0 0 0 )}$ \\
\hline CsCbGsGdDsD & $0.007(0.000)$ & $0.595(0.000)$ \\
\hline CsCbGsGuDsD & $0.008(0.000)$ & $0.619(0.000)$ \\
\hline CsCdGsGaDsD & $\mathbf{0 . 0 2 7}(\mathbf{0 . 0 0 0})$ & $\mathbf{0 . 7 7 9}(\mathbf{0 . 0 0 0})$ \\
\hline CsCdGsGdDsD & $0.011(0.000)$ & $0.615(0.000)$ \\
\hline CsCdGsGuDsD & $0.016(0.000)$ & $0.632(0.000)$ \\
\hline CsCuGsGaDsD & $\mathbf{0 . 0 2 8 ( 0 . 0 0 1 )}$ & $\mathbf{0 . 7 8 0 ( 0 . 0 0 0 )}$ \\
\hline CsCuGsGdDsD & $0.013(0.000)$ & $0.623(0.000)$ \\
\hline CsCuGsGuDsD & $0.013(0.000)$ & $0.624(0.000)$ \\
\hline CsCbGsGeAIDsD & $\mathbf{0 . 0 1 1 ( 0 . 0 0 0 )}$ & $\mathbf{0 . 7 3 9 ( 0 . 0 0 1 )}$ \\
\hline CsCbGsGdAIDsD & $0.009(0.000)$ & $0.655(0.001)$ \\
\hline CsCbGsGuAIDsD & $0.011(0.000)$ & $0.690(0.001)$ \\
\hline CsCdGsGeAIDsD & $0.013(0.000)$ & $0.709(0.000)$ \\
\hline CsCdGsGdAIDsD & $0.011(0.000)$ & $0.656(0.001)$ \\
\hline CsCdGsGuAIDsD & $0.015(0.000)$ & $0.666(0.000)$ \\
\hline CsCuGsGeAIDsD & $0.013(0.000)$ & $0.716(0.000)$ \\
\hline CsCuGsGdAIDsD & $0.012(0.000)$ & $0.660(0.000)$ \\
\hline CsCuGsGuAIDsD & $0.012(0.000)$ & $0.661(0.000)$ \\
\hline
\end{tabular}

Finally, we define the probabilities of drug efficacies along transitive paths as the normalized products of the probability matrices for individual path segments. For instance, the matrix of drugdisease associations along the path CseCbGiGaDpsD (Compound-causes-Side Effectcauses-Compound-binds-Gene-interacts-Geneassociates-Disease-presents-Symptom-presentsDisease) represents the normalized product of the probability matrices computed along the paths CseCbGiG and GiGaDpsD.

While multiple network patterns provide different clues into therapeutic effects of different drugs on different diseases, accounting for all possible paths is computationally expensive (see section 2.4). One way to lower the cost of our algorithm is to merge selected paths into paths that traverse new edges, those corresponding to the overall drug, disease and gene similarity relationships (denoted by $C s C, D s D$, and $G s G$, respectively). We define the mean drug similarity score as the average of the similarity scores based on side-effect and pharmacological profiles, or, at the matrix level, $\mathrm{CsC}=(\mathrm{CseC}+\mathrm{CpcC}) / 2$. In a similar way, we compute the average

similarity of diseases as $D s D=(D p s D+D l a D) / 2$ and the average similarity of genes as $G s G=$ $(G b p G+G p w G+G m f G+G c c G+G i G+G c G+G r G) / 7$. The notion of average similarity helps reduce the total number of paths explored by AeoNet. For instance, using the average compound similarity measure, the paths CseCtDlaD and CpcCtDlaD are merged into a single path CsCtDlaD. Table 2 shows the collection of all paths through the network explored by AeoNet.

\subsection{Measuring contribution of paths supporting drug efficacy}

Different network paths have different potentials in distinguishing disease treatments from nontreatments. In general, we have less confidence in drug-disease association scores computed along longer paths or paths that traverse meta-edges derived from sparse databases of crosslayer relationships. The treatment potential of different network patterns is computed using cross validation on the set of HetioNet drug-disease relationships (Compound-treats-Disease). Table 2 shows cross-validated AUC, AUPR and PREC@10 scores corresponding to different network patterns. 


\subsection{Aggregating drug-disease probabilities along the most informative paths}

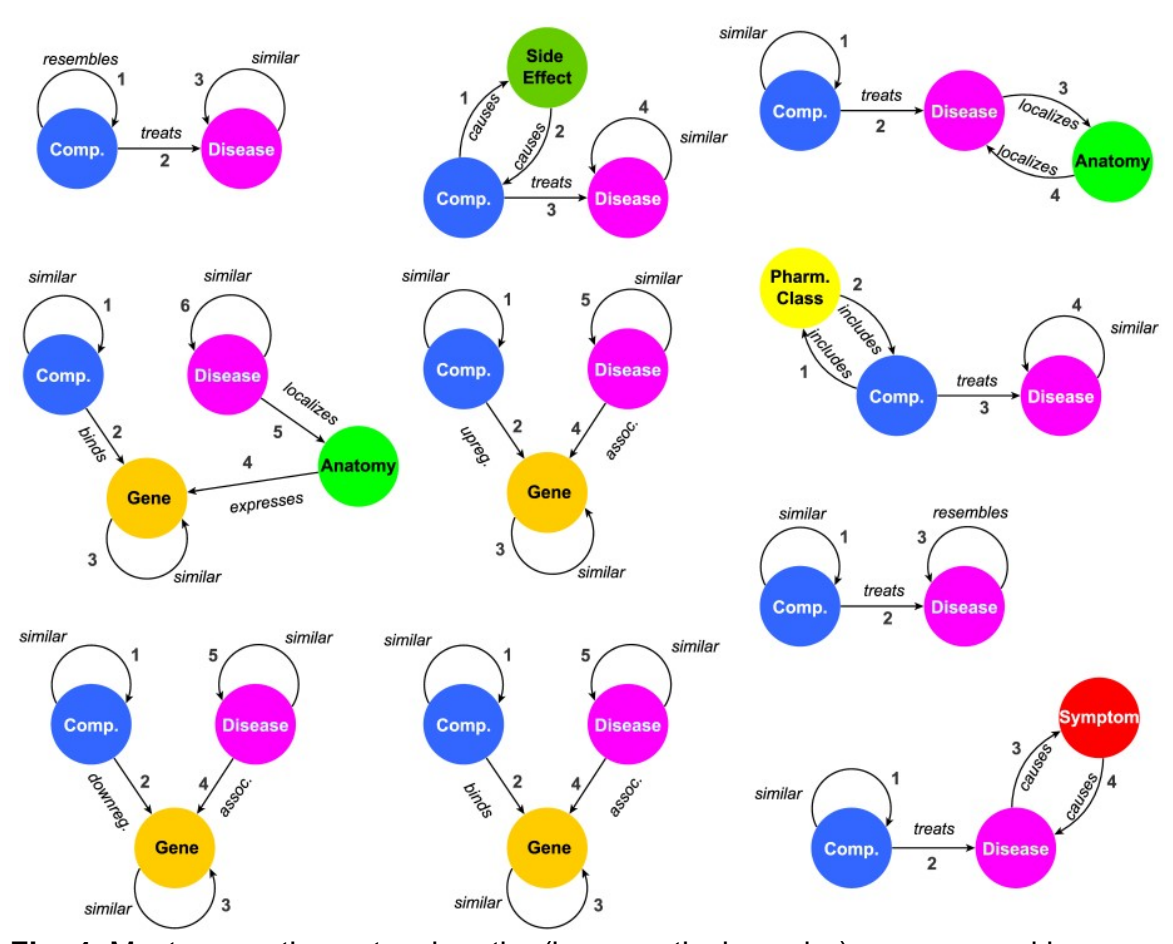

Fig. 4. Most supportive network paths (in no particular order) as measured by cross-validated AUC score.
AeoNet integrates probabilities along the network paths with highest cross-validated AUC (Table 2, Fig. 4). We note that nine out of ten highest scoring AUC paths also represent the highest scoring paths with respect to the AUPR measure, indicating a good agreement between the two classification metrics on the set of 755 indications (Compound-treatsDisease).

An optimal weighted sum of drugdisease probabilities along ten highest scoring paths is found

by maximizing cross-validated AUC score achieved on the set of Compound-treats-Disease associations. The path coefficients are selected from $C=\left\{3^{0}, 3^{1}, 3^{2}, 3^{3}\right\}$ using grid search. More specifically, for each among $4^{10}$ permutations with repetitions of 10 coefficients selected from $C$, we compute cross-validated AUC of the weighted sum of path specific probabilities and then pick the set of 10 coefficients that yields the highest AUC.

While a larger selection of network paths combined with a finer grid search has potential to improve the method's accuracy further, we had to scale both numbers down in order to balance the computational cost with the available computing resources. For predicting palliative (as opposed to disease-modifying) potentials of drugs, we exclude paths that traverse Compound-treats-Disease edges.

\section{Results}

We assess the potential of different network paths to distinguish treatments from non-treatments and the overall accuracy of AeoNe using three external validation sets compiled by Himmelstein et al., namely DrugCentral, Clinical Trials and Symptomatic. The DrugCentral test set consists of 208 novel treatments and 207,572 non-treatments extracted from the DrugCentral repository ${ }^{40}$. The Clinical Trial set consists of 5,594 novel indications compiled from ClinicalTrials.gov and 202,186 non-treatments. We note that, while much larger, the Clinical Trial benchmark is less reliable than DrugCentral, due to the low approval rates of drugs in clinical trials. The Symptomatic set contains 390 HetioNet relationships (positive associations) of the type Compound-palliates-Disease ${ }^{14}$ and 208,023 negative associations. We emphasize that no positive drug-disease association from any test sets appears in our training set of 755 HetioNet disease-modifying indications (Compound-treats-Disease relationships). 
Table 3 list the classification scores along the individual network paths and the scores obtained using the integrative AeoNet approach. As seen in this table, the value added by different network paths varies across the three test sets. For instance, the path with the highest AUPR score in the DrugCentral benchmark is CpcCtDsD (AUPR $=0.095$ ) but this path does not perform nearly as well in other benchmarks (it ranks \#6 in DrugCentral AUC benchmark and \#6 and \#8 in Clinical Trial AUPR and AUC benchmarks, respectively). On the other hand, the path $C s C b G s G a D s D$ has the highest $A U C$ score $(A U C=0.747)$ and the second highest AUPR score (AUPR $=0.097)$ in Clinical Trial benchmark but it ranks in the bottom half of all paths in the DrugCentral benchmarks with respect to both AUC and AUPR. By finding and exploiting synergies between different network paths, AeoNet consistently improves classification scores of individual network paths (Table 3).

Table 3. Classification accuracy along individual network paths and the accuracy of the integrative approach. Highest scoring individual paths are denoted by *. Best method overall is shown in bold.

\begin{tabular}{l|l|l|l|l}
\hline & \multicolumn{2}{|l|}{ DrugCentral } & \multicolumn{2}{c}{ Clinical Trial } \\
\hline Method & AUPR & AUC & AUPR & AUC \\
\hline$C r C t D s D$ & 0.058 & 0.857 & 0.075 & 0.698 \\
\hline$C p c C t D s D$ & $0.095^{*}$ & 0.817 & 0.080 & 0.677 \\
\hline$C s e C t D s D$ & 0.048 & 0.842 & $0.110^{*}$ & 0.720 \\
\hline$C s C t D r D$ & 0.083 & 0.858 & 0.090 & 0.693 \\
\hline CsCtDlaD & 0.083 & 0.862 & 0.089 & 0.698 \\
\hline CsCtDpsD & 0.083 & $0.863^{*}$ & 0.088 & 0.696 \\
\hline CsCbGsGaDsD & 0.006 & 0.748 & 0.097 & $0.747^{*}$ \\
\hline CsCdGsGuDsD & 0.002 & 0.657 & 0.072 & 0.669 \\
\hline CsCuGsGdDsD & 0.003 & 0.664 & 0.067 & 0.670 \\
\hline CsCbGsGeAaDsD & 0.001 & 0.584 & 0.074 & 0.721 \\
\hline AeoNet & $\mathbf{0 . 1 0 3}$ & $\mathbf{0 . 8 9 2}$ & $\mathbf{0 . 1 2 2}$ & $\mathbf{0 . 7 9 5}$ \\
\hline
\end{tabular}

We also present a side-by-side comparison of AeoNet with Rephetio and two other recently published methods for predicting drug-associated indications, BNNR ${ }^{60}$ and $\mathrm{OMC}^{61}$. The last two algorithms have been shown to yield higher accuracy when compared to other state-of-the-art approaches. These methods require drug similarity information in terms of Tanimoto coefficients as well as the disease similarity information. We computed Tanimoto coefficients by calculating Jaccard similarity between the drug fingerprints derived from the Chemistry Development Kit, using PaDEL-Descriptor

software ${ }^{62}$. For disease side information, both BNNR and OMC were given Disease-resemblesDisease scores. In addition, the OMC method tested here is based on tri-layer network (OMC3), and it requires drug-gene and disease-gene association matrices as input. We derived this data from HetioNet's Drug-binds-Gene and Disease-associates-Gene relationships, respectively. All three external methods were ran using the default parameters supplied by their authors.

With the exception of Clinical Trial PREC@10 test (OMC 40\%, AeoNet 40\%), the classification scores achieved by AeoNet exceed the scores achieved by the other methods in every benchmark and according to each classification measure (Table 3, Fig. 5). The performance of the remaining three methods fluctuate across the test sets and across different benchmarking measures used. For instance, Rephetio has the second highest AUC scores in every benchmark but all other methods compare favorably to Rephetio in PREC@10 tests. The AUC scores achieved by OMC trail those achieved by AeoNet and Rephetio but this method shows a consistently good performance in AUPR and PREC@10 tests.

The superior performance of our method in recovering Symptomatic relationships is suggestive of different action mechanisms of disease-modifying therapies compared to palliative ones. We re-emphasize that AeoNet computes probabilities of palliative therapies along the transitive paths from drugs to diseases (those that pass through the gene nodes) ignoring all paths that include Compound-treats-Disease edge.

The seemingly low AUPR scores of the four methods in the three benchmark need proper interpretation. To place the AUPR scores in context, we compare them to the scores obtained by a purely random classifier. The AUPR score achieved by a random classifier is equal to the fraction of condition positives in the test set $\left(\sum\right.$ cond.pos / $\left(\sum\right.$ cond.pos + $\sum$ cond.neg)). For instance, the AUPR score achieved by a random classifier on DrugCentral 
data set is $\sim 0.001$ (208 condition positives and 207,572 condition negatives). For comparison, on the same data set the AeoNet method achieves the AUPR score of 0.103 , which represents two orders of magnitude (100 fold) enrichment over the random classifier.
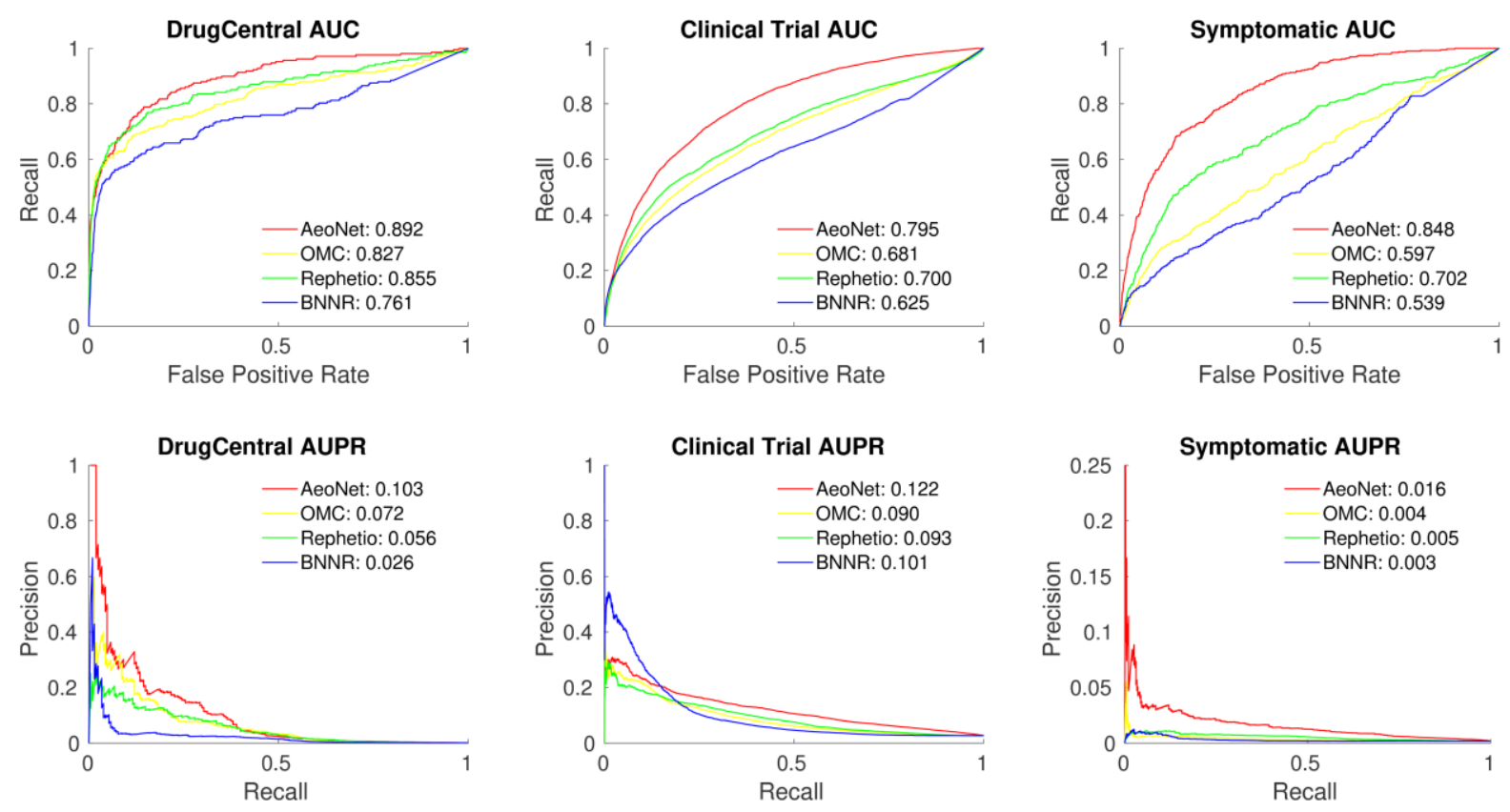

DrugCentral PREC@10

Clinical Trial PREC@10

Symptomatic PREC@10
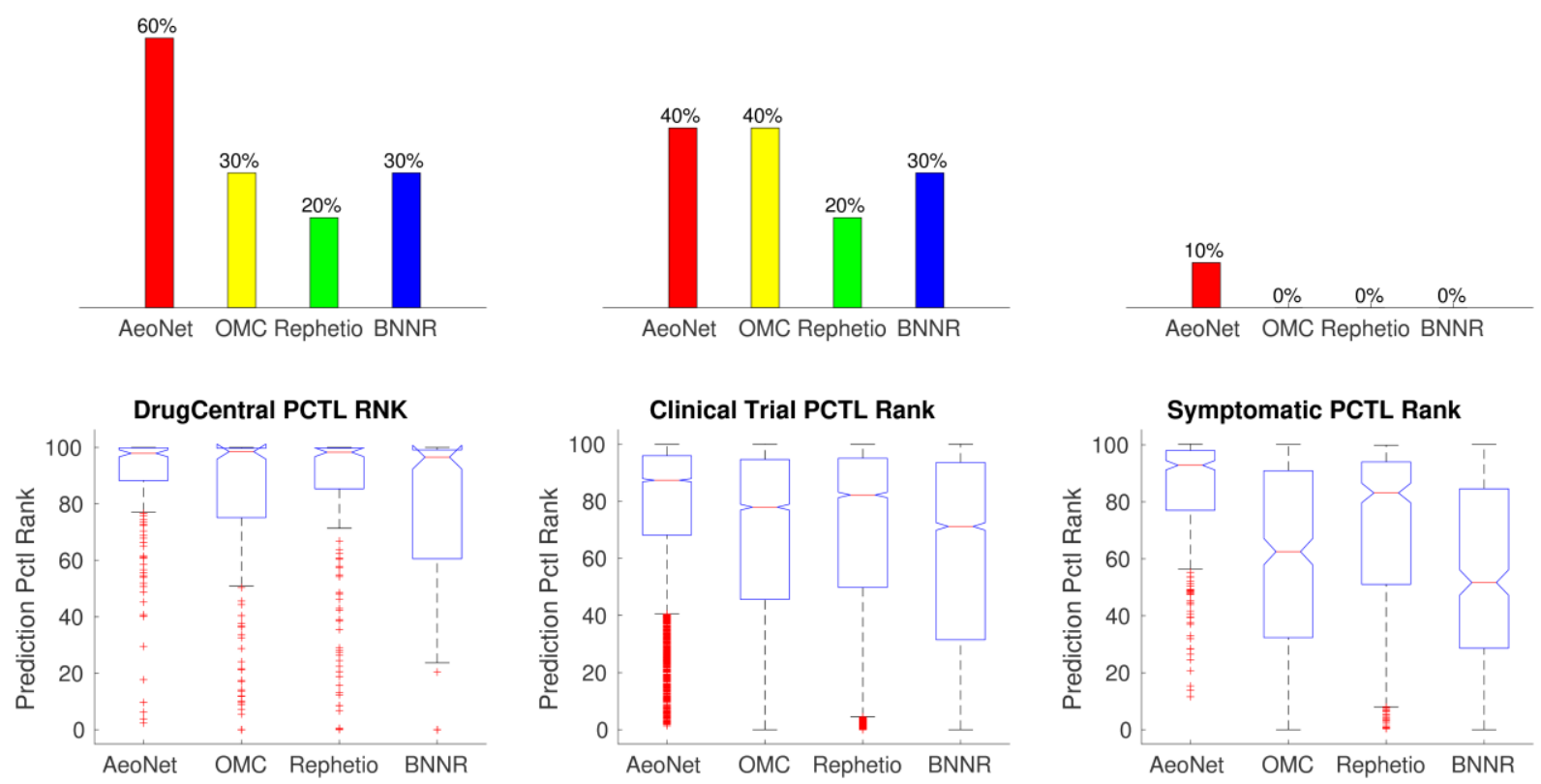

Fig. 5. Comparing AeoNet with state-of-art using different classification metrics: AUPR, AUC, PREC@10, and Prediction Percentile Rank.

We observe that, at any p-value cutoff, the fraction of DrugCentral indications recovered by AeoNet is much larger compared to the percentage of recovered Clinical Trial indications 
(Fig 6). This is expected and desirable, as a majority of Clinical Trial drugs fail to get FDA approval. In fact, the recovery rate of Clinical Trial indications exceeding the FDA drug approval rate (currently around $9.6 \%{ }^{63}$ ) should be indicative of poor method's specificity.
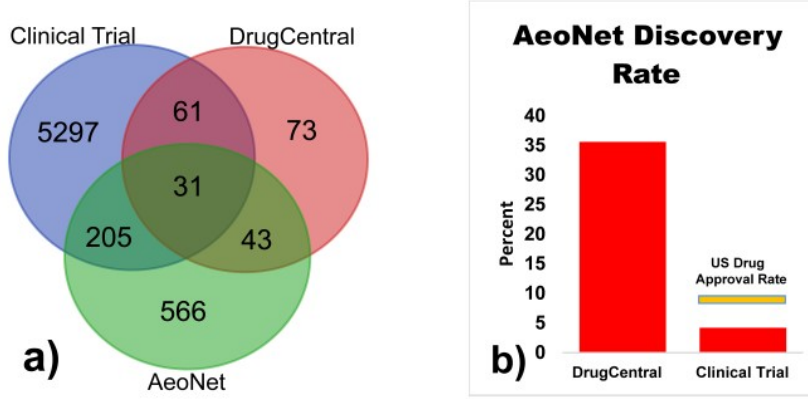

Fig 6. (a) The overlap of 845 AeoNet predictions with pvalues $\leq 1.0 \mathrm{E}-3$ with those stored in DrugCentral and Clinical Trial (training set indications not included) (b) Percentage of DrugCentral and Clinical Trial indications found by AeoNet using the same p-vaue cutoff. Current FDA drug approval rate is shown in orange.

\begin{abstract}
Illustrative example - Bone Cancer
Dactinomycin is an intravenous therapy indicated for Ewing's sarcoma, a type of cancer that grows in bone or surrounding tissues, mostly in children and young adults. Dactinomycin represents a good repurposing test for AeoNet, as its association with bone-cancer is listed in DrugCentral but not in HetioNet.
\end{abstract}

The top six AeoNet predictions for the treatment of bone cancer are Epirubicin, Cisplatin, Methotrexate, Carboplatin, Doxorubicin, and Dactinomycin (in ranked order). We note that the first five hits are relatively easy cases for AeoNet, because the associations of those drugs with bone-cancer are already recorded in HetioNet. AeoNet places Dactinomycin ( $p$-value=2.2E-8) on top of all drugs that are not listed as one of 755 HetioNet diseasemodifying therapies for bone-cancer, despite the fact that the drug lacks structural analogs among drugs with similar indications in HetioNet. In fact, the closest structural neighbor of Dactinomycin in HetioNet, as measured by Tanimoto coefficient, is Moxifloxacin, an antibiotic used to treat bacterial infections. Moreover, a closer inspection of Compound-resembles-Compound relationships reveals that no drug "resembles" Dactinomycin in HetioNet, further illustrating AeoNet's ability to make accurate inferences based on aggregate probabilities of different transitive network paths.

\section{Illustrative example - Gout}

Gout is a general term for different conditions caused by the deposits of monosodium urate crystals, typically in the joints of the foot or ankle. There are six treatments of gout in DrugCentral that are not present in HetioNet. We wanted to find out whether AeoNet can confidently predict any of those six drugs as potential repurposing candidates.

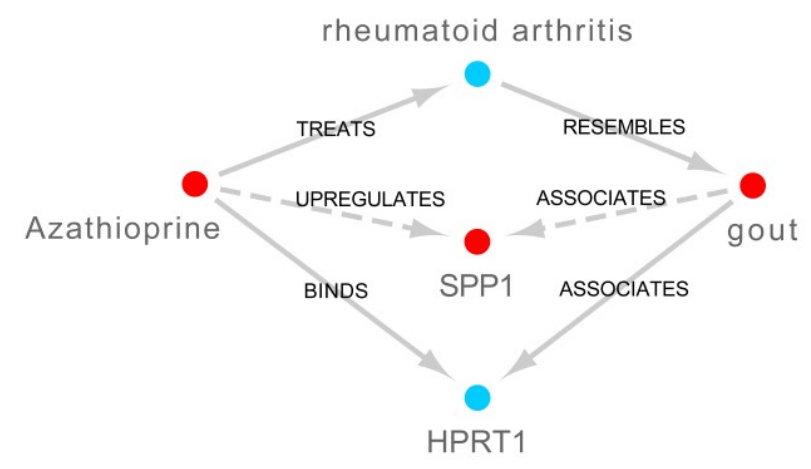

Fig. 7. Most significant paths of length 2 connecting Azathioprine and gout. Solid edges represent existing HetioNet relationships. Dashed edges (forming a new network path) are predicted by compressed sensing.
After excluding gout associations recorded in HetioNet (which are consistently found in the highest ranks by our method) the top eight AeoNet predictions for gout treatments include four drugs listed in DrugCentral but not HetioNet, namely Triamcinolone, Dexamethasone, Betamethasone and Azathioprine. While the first three hits have p-values below 1.0E-13 and despite these drugs not being present in our training set, we still consider them relatively easy predictions, as each one of those drugs has a close chemical analog in HetioNet. However, Azathioprine ( $\mathrm{p}$-value $=2.0 \mathrm{E}-4)$ is a difficult prediction case since a closer inspection of Compound-resembles-Compound relationships reveals that Azathioprine does not resemble any drug with known HetioNet indications. Moreover, Azathioprine is not structurally similar to any other gout treatment in 
HetioNet (Tanimoto coef. < 0.27). Fig. 7 shows three strong Azathioprine-goat supporting paths identified by AeoNet, including a path that span two novel edges that do not appear in HetioNet, namely Azathioprine upregulates-SPP1 (predicted p-value: 4.3E-12) and gout-associates-SPP1 (p-value: $8.9 \mathrm{E}-16)^{64}$.

\section{Discussion and Conclusion}

Heterogeneous networks are emerging as a powerful way to model complex biological systems. Network implementations allow the inference of relationships among distantly related biological entities and improve the prediction of associations between closely related entities. We present a computational technique for finding potential drug repurposing candidates from a comprehensive network of interactions between tens of thousands of biomedical entities, including drugs, side-effects, diseases, symptoms, and genes. Our methodology for distinguishing disease treatments from non-treatments integrates the likelihoods of different network patterns supporting drug efficacy. In contrast to previous studies that seek to explore only experimentally verified relationships, the method we propose operates on a much larger, probabilistic network that consists of tens of millions of predicted relationships between the network nodes. With minor modifications, our algorithm can be used in other inference tasks, such as drug-target interaction or disease-gene association prediction.

The results of our study hint at some straightforward network enhancement that can further increase the prediction accuracy. For instance, expanding the network to include compound structural similarity, gene homology and/or gene-side effect associations ${ }^{65}$ would enrich the set of paths supporting drug efficacy and, in turn, provide further insights into drug mechanisms of actions. These modifications, along with the fast growing biomedical databases and advances in machine learning algorithms will fuel research into computational drug discovery and repurposing in the years to come.

\section{References}

1. Scannell, J. W., Blanckley, A., Boldon, H., \& Warrington, B. (2012). Diagnosing the decline in pharmaceutical R\&D efficiency. Nature reviews Drug discovery, 11(3), 191.

2. Collier R. Drug development cost estimates hard to swallow. CMAJ. 2009 Feb 3;180(3):279-80.

3. Hopkins, A. L. (2008). Network pharmacology: the next paradigm in drug discovery. Nature chemical biology, 4(11), 682.

4. Hopkins, A. L. (2007). Network pharmacology. Nature biotechnology, 25(10), 1110-1111.

5. Lee, B., Zhang, S., Poleksic, A., \& Xie, L. (2020). Heterogeneous Multi-Layered Network Model for Omics Data Integration and Analysis. Frontiers in Genetics, 10, 1381.

6. Oprea, T. I., Nielsen, S. K., Ursu, O., Yang, J. J., Taboureau, O., Mathias, S. L., ... \& Bologa, C. G. (2011). Associating drugs, targets and clinical outcomes into an integrated network affords a new platform for computer-aided drug repurposing. Molecular informatics, 30(2-3), 100-111.

7. Vitali, F., Cohen, L. D., Demartini, A., Amato, A., Eterno, V., Zambelli, A., \& Bellazzi, R. (2016). A network-based data integration approach to support drug repurposing and multitarget therapies in triple negative breast cancer. PloS one, 11(9).

8. Pujol, A., Mosca, R., Farrés, J., \& Aloy, P. (2010). Unveiling the role of network and systems biology in drug discovery. Trends in pharmacological sciences, 31(3), 115-123. 
9. Luo, Y., Zhao, X., Zhou, J., Yang, J., Zhang, Y., Kuang, W., ... \& Zeng, J. (2017). A network integration approach for drug-target interaction prediction and computational drug repositioning from heterogeneous information. Nature communications, 8(1), 1-13.

10. Zeng, X., Zhu, S., Liu, X., Zhou, Y., Nussinov, R., \& Cheng, F. (2019). deepDR: a networkbased deep learning approach to in silico drug repositioning. Bioinformatics, 35(24), 51915198.

11. Chen, C., Tong, H., Xie, L., Ying, L., \& He, Q. (2016, August). FASCINATE: fast cross-layer dependency inference on multi-layered networks. In Proceedings of the 22nd ACM SIGKDD International Conference on Knowledge Discovery and Data Mining (pp. 765-774).

12. Guney, E., Menche, J., Vidal, M., \& Barábasi, A. L. (2016). Network-based in silico drug efficacy screening. Nature communications, 7, 10331.

13. Cheng, F., Desai, R. J., Handy, D. E., Wang, R., Schneeweiss, S., Barabási, A. L., \& Loscalzo, J. (2018). Network-based approach to prediction and population-based validation of in silico drug repurposing. Nature communications, 9(1), 1-12.

14. Himmelstein, D. S., Lizee, A., Hessler, C., Brueggeman, L., Chen, S. L., Hadley, D., ... \& Baranzini, S. E. (2017). Systematic integration of biomedical knowledge prioritizes drugs for repurposing. Elife, 6, e26726.

15. Law, V., Knox, C., Djoumbou, Y., Jewison, T., Guo, A. C., Liu, Y., ... \& Tang, A. (2014). DrugBank 4.0: shedding new light on drug metabolism. Nucleic acids research, 42(D1), D1091-D1097.

16. Kibbe, W. A., Arze, C., Felix, V., Mitraka, E., Bolton, E., Fu, G., ... \& Parkinson, H. (2015). Disease Ontology 2015 update: an expanded and updated database of human diseases for linking biomedical knowledge through disease data. Nucleic acids research, 43(D1), D1071D1078.

17. Schriml, L. M., Arze, C., Nadendla, S., Chang, Y. W. W., Mazaitis, M., Felix, V., ... \& Kibbe, W. A. (2012). Disease Ontology: a backbone for disease semantic integration. Nucleic acids research, 40(D1), D940-D946.

18. Maglott, D., Ostell, J., Pruitt, K. D., \& Tatusova, T. (2010). Entrez Gene: gene-centered information at NCBI. Nucleic acids research, 39(suppl_1), D52-D57.

19. Kuhn, M., Letunic, I., Jensen, L. J., \& Bork, P. (2016). The SIDER database of drugs and side effects. Nucleic acids research, 44(D1), D1075-D1079.

20. US National Library of Medicine. Fact Sheet: Medline. Bethesda, MD: U.S. National Library of Medicine, 2004 (updated May 7, 2014). http://www.nlm.nih.gov/pubs/factsheets/medline.html.

21. Kutmon, M., Riutta, A., Nunes, N., Hanspers, K., Willighagen, E. L., Bohler, A., ... \& Coort, S. L. (2016). WikiPathways: capturing the full diversity of pathway knowledge. Nucleic acids research, 44(D1), D488-D494.

22. Pico, A. R., Kelder, T., Van lersel, M. P., Hanspers, K., Conklin, B. R., \& Evelo, C. (2008). WikiPathways: pathway editing for the people. PLoS biology, 6(7).

23. Fabregat, A., Sidiropoulos, K., Garapati, P., Gillespie, M., Hausmann, K., Haw, R., ... \& Matthews, L. (2016). The reactome pathway knowledgebase. Nucleic acids research, 44(D1), D481-D487. 
24. Schaefer, C. F., Anthony, K., Krupa, S., Buchoff, J., Day, M., Hannay, T., \& Buetow, K. H. (2009). PID: the pathway interaction database. Nucleic acids research, 37(suppl_1), D674D679.

25. Cerami, E. G., Gross, B. E., Demir, E., Rodchenkov, I., Babur, Ö., Anwar, N., ... \& Sander, C. (2010). Pathway Commons, a web resource for biological pathway data. Nucleic acids research, 39(suppl_1), D685-D690.

26. Ashburner, M., Ball, C. A., Blake, J. A., Botstein, D., Butler, H., Cherry, J. M., ... \& Harris, M. A. (2000). Gene ontology: tool for the unification of biology. Nature genetics, 25(1), 25-29.

27. Mungall, C. J., Torniai, C., Gkoutos, G. V., Lewis, S. E., \& Haendel, M. A. (2012). Uberon, an integrative multi-species anatomy ontology. Genome biology, 13(1), R5.

28. Ursu, O., Holmes, J., Knockel, J., Bologa, C. G., Yang, J. J., Mathias, S. L., ... \& Oprea, T. I. (2016). DrugCentral: online drug compendium. Nucleic acids research, gkw993.

29. Khare, R., Burger, J. D., Aberdeen, J. S., Tresner-Kirsch, D. W., Corrales, T. J., Hirchman, L., \& Lu, Z. (2015). Scaling drug indication curation through crowdsourcing. Database, 2015.

30. Khare, R., Li, J., \& Lu, Z. (2014). LabeledIn: cataloging labeled indications for human drugs. Journal of biomedical informatics, 52, 448-456.

31. Wei, W. Q., Cronin, R. M., Xu, H., Lasko, T. A., Bastarache, L., \& Denny, J. C. (2013). Development and evaluation of an ensemble resource linking medications to their indications. Journal of the American Medical Informatics Association, 20(5), 954-961.

32. Gottlieb, A., Stein, G. Y., Ruppin, E., \& Sharan, R. (2011). PREDICT: a method for inferring novel drug indications with application to personalized medicine. Molecular systems biology, 7(1).

33. McCoy, A. B., Wright, A., Laxmisan, A., Ottosen, M. J., McCoy, J. A., Butten, D., \& Sittig, D. F. (2012). Development and evaluation of a crowdsourcing methodology for knowledge base construction: identifying relationships between clinical problems and medications. Journal of the American Medical Informatics Association, 19(5), 713-718.

34. Bastian, F., Parmentier, G., Roux, J., Moretti, S., Laudet, V., \& Robinson-Rechavi, M. (2008). Data Integration in the Life Sciences. Lecture Notes in Computer Science, 124-131.

35. Santos, A., Tsafou, K., Stolte, C., Pletscher-Frankild, S., O’Donoghue, S. I., \& Jensen, L. J. (2015). Comprehensive comparison of large-scale tissue expression datasets. PeerJ, 3, e1054.

36. Chen, X., Liu, M., \& Gilson, M. K. (2001). BindingDB: a web-accessible molecular recognition database. Combinatorial chemistry \& high throughput screening, 4(8), 719-725.

37. Gilson, M. K., Liu, T., Baitaluk, M., Nicola, G., Hwang, L., \& Chong, J. (2016). BindingDB in 2015: a public database for medicinal chemistry, computational chemistry and systems pharmacology. Nucleic acids research, 44(D1), D1045-D1053.

38. Law, V., Knox, C., Djoumbou, Y., Jewison, T., Guo, A. C., Liu, Y., ... \& Tang, A. (2014). DrugBank 4.0: shedding new light on drug metabolism. Nucleic acids research, 42(D1), D1091-D1097.

39. Wishart, D. S., Knox, C., Guo, A. C., Shrivastava, S., Hassanali, M., Stothard, P., ... \& Woolsey, J. (2006). DrugBank: a comprehensive resource for in silico drug discovery and exploration. Nucleic acids research, 34(suppl_1), D668-D672. 
40. Ursu, O., Holmes, J., Knockel, J., Bologa, C. G., Yang, J. J., Mathias, S. L., ... \& Oprea, T. I. (2016). DrugCentral: online drug compendium. Nucleic acids research, gkw993.

41. Subramanian, A., Narayan, R., Corsello, S. M., Peck, D. D., Natoli, T. E., Lu, X., ... \& Lahr, D. L. (2017). A next generation connectivity map: L1000 platform and the first 1,000,000 profiles. Cell, 171(6), 1437-1452.

42. MacArthur, J., Bowler, E., Cerezo, M., Gil, L., Hall, P., Hastings, E., ... \& Pendlington, Z. M. (2017). The new NHGRI-EBI Catalog of published genome-wide association studies (GWAS Catalog). Nucleic acids research, 45(D1), D896-D901.

43. Pletscher-Frankild, S., Pallejà, A., Tsafou, K., Binder, J. X., \& Jensen, L. J. (2015). DISEASES: Text mining and data integration of disease-gene associations. Methods, 74, 83-89.

44. Piñero, J., Bravo, À., Queralt-Rosinach, N., Gutiérrez-Sacristán, A., Deu-Pons, J., Centeno, E., ... \& Furlong, L. I. (2016). DisGeNET: a comprehensive platform integrating information on human disease-associated genes and variants. Nucleic acids research, gkw943.

45. Piñero, J., Queralt-Rosinach, N., Bravo, A., Deu-Pons, J., Bauer-Mehren, A., Baron, M., ... \& Furlong, L. I. (2015). DisGeNET: a discovery platform for the dynamical exploration of human diseases and their genes. Database, 2015.

46. Xu, W., Wang, H., Cheng, W., Fu, D., Xia, T., Kibbe, W. A., \& Lin, S. M. (2012). A framework for annotating human genome in disease context. PLoS One, 7(12).

47. Priedigkeit, N., Wolfe, N., \& Clark, N. L. (2015). Evolutionary signatures amongst disease genes permit novel methods for gene prioritization and construction of informative genebased networks. PLoS genetics, 11(2).

48. Rual, J. F., Venkatesan, K., Hao, T., Hirozane-Kishikawa, T., Dricot, A., Li, N., ... \& Klitgord, N. (2005). Towards a proteome-scale map of the human protein-protein interaction network. Nature, 437(7062), 1173-1178.

49. Venkatesan, K., Rual, J. F., Vazquez, A., StelzI, U., Lemmens, I., Hirozane-Kishikawa, T., ... \& Yildirim, M. A. (2009). An empirical framework for binary interactome mapping. Nature methods, 6(1), 83.

50. Yu, H., Tardivo, L., Tam, S., Weiner, E., Gebreab, F., Fan, C., ... \& Sahalie, J. (2011). Nextgeneration sequencing to generate interactome datasets. Nature methods, 8(6), 478.

51. Rolland, T., Taşan, M., Charloteaux, B., Pevzner, S. J., Zhong, Q., Sahni, N., ... \& Kamburov, A. (2014). A proteome-scale map of the human interactome network. Cell, 159(5), 1212-1226.

52. Menche, J., Sharma, A., Kitsak, M., Ghiassian, S. D., Vidal, M., Loscalzo, J., \& Barabási, A. L. (2015). Uncovering disease-disease relationships through the incomplete interactome. Science, 347(6224), 1257601.

53. Himmelstein, D. S., \& Baranzini, S. E. (2015). Heterogeneous network edge prediction: a data integration approach to prioritize disease-associated genes. PLoS computational biology, 11(7).

54. Huntley, R. P., Sawford, T., Mutowo-Meullenet, P., Shypitsyna, A., Bonilla, C., Martin, M. J., \& O'Donovan, C. (2015). The GOA database: gene ontology annotation updates for 2015. Nucleic acids research, 43(D1), D1057-D1063. 
55. Lim, H., Gray, P., Xie, L., \& Poleksic, A. (2016). Improved genome-scale multi-target virtual screening via a novel collaborative filtering approach to cold-start problem. Scientific reports, 6(1), 1-11.

56. Poleksic, A., \& Xie, L. (2018). Predicting serious rare adverse reactions of novel chemicals. Bioinformatics, 34(16), 2835-2842.

57. Lim, H., Poleksic, A., Yao, Y., Tong, H., He, D., Zhuang, L., ... \& Xie, L. (2016). Large-scale off-target identification using fast and accurate dual regularized one-class collaborative filtering and its application to drug repurposing. PLoS computational biology, 12(10), e1005135.

58. Yao, Y., Tong, H., Yan, G., Xu, F., Zhang, X., Szymanski, B. K., \& Lu, J. (2014, November). Dual-regularized one-class collaborative filtering. In Proceedings of the 23rd ACM International Conference on Conference on Information and Knowledge Management (pp. 759-768).

59. Steck, H. (2010, July). Training and testing of recommender systems on data missing not at random. In Proceedings of the 16th ACM SIGKDD international conference on Knowledge discovery and data mining (pp. 713-722).

60. Yang, M., Luo, H., Li, Y., \& Wang, J. (2019). Drug repositioning based on bounded nuclear norm regularization. Bioinformatics, 35(14), i455-i463.

61. Yang, M., Luo, H., Li, Y., \& Wang, J. (2019). Drug repositioning based on bounded nuclear norm regularization. Bioinformatics, 35(14), i455-i463.

62. Yap, C. W. (2011). PaDEL-descriptor: An open source software to calculate molecular descriptors and fingerprints. Journal of computational chemistry, 32(7), 1466-1474.

63. Thomas, D. W., Burns, J., Audette, J., Carroll, A., Dow-Hygelund, C., \& Hay, M. (2016). Clinical development success rates 2006-2015. BIO Industry Analysis, 1, 16.

64. Chen, C. J., Tseng, C. C., Yen, J. H., Chang, J. G., Chou, W. C., Chu, H. W., ... \& Liao, W. T. (2018). ABCG2 contributes to the development of gout and hyperuricemia in a genomewide association study. Scientific reports, 8(1), 1-9.

65. Ji, Z. L., Han, L. Y., Yap, C. W., Sun, L. Z., Chen, X., \& Chen, Y. Z. (2003). Drug adverse reaction target database (DART). Drug safety, 26(10), 685-690. 
bioRxiv preprint doi: https://doi.org/10.1101/2020.06.07.138966; this version posted June 8, 2020. The copyright holder for this preprint (which was not certified by peer review) is the author/funder, who has granted bioRxiv a license to display the preprint in perpetuity. It is made available under aCC-BY-NC 4.0 International license.

Acknowledgements: We are thankful to Prof. Lei Xie from CUNY Graduate Center for inspiring discussions on the topic. We also thank Daniel Himmelstein for helping us compile the three external validation sets. This work is supported in part by the University of Northern lowa Professional Development award, the Capacity Building grant and the Google Cloud grant. 